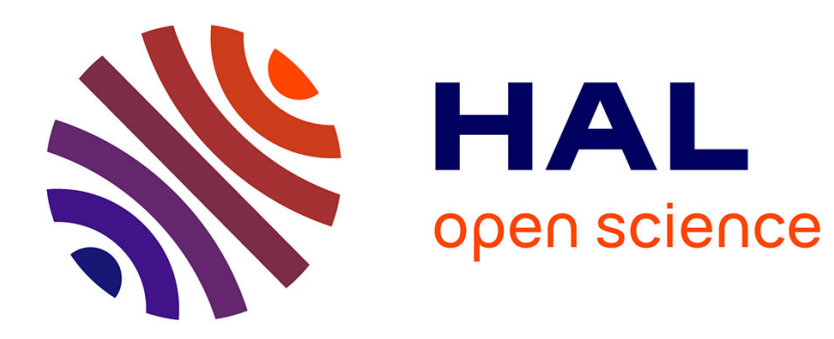

\title{
A novel approach to dynamic soaring modeling and simulation
}

\author{
Jean-Marie Kai, Tarek Hamel, Claude Samson
}

\section{To cite this version:}

Jean-Marie Kai, Tarek Hamel, Claude Samson. A novel approach to dynamic soaring modeling and simulation. 2020. hal-02481563

\section{HAL Id: hal-02481563 \\ https://hal.science/hal-02481563}

Preprint submitted on 17 Feb 2020

HAL is a multi-disciplinary open access archive for the deposit and dissemination of scientific research documents, whether they are published or not. The documents may come from teaching and research institutions in France or abroad, or from public or private research centers.
L'archive ouverte pluridisciplinaire HAL, est destinée au dépôt et à la diffusion de documents scientifiques de niveau recherche, publiés ou non, émanant des établissements d'enseignement et de recherche français ou étrangers, des laboratoires publics ou privés. 


\title{
A novel approach to dynamic soaring
}

\section{modeling and simulation}

\author{
Jean-Marie $\mathrm{Kai}^{1}$ and Tarek $\mathrm{Hamel}^{2}$ \\ Laboratoire I3S-CNRS, Université Côte d'Azur (UCA), Sophia Antipolis, 06900, France
}

\author{
Claude Samson ${ }^{3}$ \\ INRIA and Laboratoire I3S-CNRS, Université Côte d'Azur (UCA), Sophia Antipolis, 06900, France
}

This paper revisits dynamic soaring on the basis of a nonlinear point-mass flight dynamics model previously used for scale-model aircraft to design path-following autopilots endowed with theoretically and experimentally demonstrated stability and convergence properties. The energy-harvesting process associated with specific maneuvers of a glider subjected to horizontal wind, and on which dynamic soaring relies, is explained at the light of this model. Expressions for the estimates of various variables involved in dynamic soaring along inclined circular paths crossing a thin wind shear layer, as experienced by model glider pilots over the world, are derived via approximate integration of the model equations. Given a glider's path and a wind profile, this model also presents the asset of yielding an explicit ordinary differential equation that entirely characterizes the time-evolution of the modeled glider's state along the path, thus allowing for an easy simulation of dynamic soaring over a large variety of operating conditions. We view this simulation facility as a tool that usefully complements other studies of dynamic soaring that focus on trajectory optimization via dynamic programming. Its usefulness is here illustrated by first validating the aforementioned estimates in the case of circular trajectories crossing a thin wind shear layer, then by showing how it applies to other examples of trajectories and ocean wind profile models

\footnotetext{
1 Ph.D. candidate, Laboratoire I3S- CNRS, Université Côte d'Azur (UCA), Sophia Antipolis, France, kai@i3s.unice.fr 2 Professor, Institut Universitaire de France and Université Côte d'Azur (UCA), Sophia Antipolis, France, thamel@i3s.unice.fr

3 Research Director, INRIA and I3S-CNRS, Université Côte d'Azur (UCA), Sophia Antipolis, France, claude.samson@inria.fr, csamson@i3s.unice.fr
} 


\section{Nomenclature}

$\mathbb{R}^{n} \quad=n$-dimensional real vector space.

$\mathcal{I}=\left\{O ; \boldsymbol{\imath}_{0}, \boldsymbol{\jmath}_{0}, \boldsymbol{k}_{0}\right\}=$ inertial frame.

$\boldsymbol{E}^{3}=3 D$ Euclidean vector space with $\mathcal{I}$ the associated reference frame.

Vectors in $\boldsymbol{E}^{3}$ are denoted with bold letters.

$|x| \quad=$ Euclidean norm of $x \in \mathbb{R}^{n}$.

$|\boldsymbol{x}|(=|x|) \quad=$ Euclidean norm of $\boldsymbol{x} \in \boldsymbol{E}^{3}$.

$x_{i}(i=1, \ldots, n)=i$ th component of $x \in \mathbb{R}^{n}$.

$P \quad=$ aircraft center of mass $(\mathrm{CoM})$

$\mathcal{B}=\{P ; \boldsymbol{\imath}, \boldsymbol{\jmath}, \boldsymbol{k}\} \quad=$ body-fixed frame with $\boldsymbol{\imath}$ taken on the zero-lift body-axis of the aircraft.

m = vehicle's mass.

$\boldsymbol{v} \quad=$ aircraft CoM's velocity with respect to $\mathcal{I}$.

$\boldsymbol{a}(=\dot{\boldsymbol{v}}) \quad=$ aircraft CoM's acceleration with respect to $\mathcal{I}$.

$\boldsymbol{g}=g_{0} \boldsymbol{k}_{0} \quad=$ gravitational acceleration

$\boldsymbol{v}_{w} \quad=$ ambient wind velocity with respect to $\mathcal{I}$.

$\boldsymbol{v}_{a}=\boldsymbol{v}-\boldsymbol{v}_{w} \quad=$ aircraft air-velocity with respect to $\mathcal{I}$.

x.y inner product of $\boldsymbol{x} \in \boldsymbol{E}^{3}$ and $\boldsymbol{y} \in \boldsymbol{E}^{3}$.

$\xi \quad=$ coordinate vector of $\boldsymbol{\xi} \in \boldsymbol{E}^{3}$ in the body-fixed frame $\mathcal{B}$,

i.e. $\xi=\left[\xi_{1}, \xi_{2}, \xi_{3}\right]^{\top}, \xi_{1}=\boldsymbol{\xi} . \boldsymbol{\imath}, \xi_{2}=\boldsymbol{\xi} . \boldsymbol{\jmath}, \xi_{3}=\boldsymbol{\xi} . \boldsymbol{k}, \boldsymbol{\xi}=\xi_{1} \boldsymbol{\imath}+\xi_{2} \boldsymbol{J}+\xi_{3} \boldsymbol{k}$.

\section{Introduction}

Since the pioneering investigation by Lord Rayleigh in 1883 [1] about the mechanisms involved in long-range flights of birds over the ocean, the possibility for a bird, or a man-made glider, of harvesting energy by repeatedly crossing a wind shear layer, in order to stay aloft for a long time, has motivated many studies. Over the years a large amount of data has been gathered by 
naturalists who observed large birds, albatrosses in particular, which mastered this type of flight. One may for instance consult $[2,3]$ for a digest of these findings. More recently the subject has also attracted the interest of engineers who analyze dynamic soaring (DS) on the basis of flight dynamics and ocean-wind profile mathematical models. Among early seminal contributions of this type one may cite $[2,4-6]$ where the authors combine observations about the flight of albatrosses with dynamics modeling equations and the support of numerical simulation to explain the principle of DS. Calculation of optimal DS trajectories via dynamic programming was initiated by Sachs [4] and became a common factor of many recent engineering-oriented DS studies [2, 3, 7-11]. The adjacent issues of sensory state estimation and control for a glider to automatically follow a desired (possibly optimal) path and/or take advantage of wind gusts have also been addressed in several studies of DS $[7,10,12-14]$. The DS research theme is concomitant with experiments conducted by enthusiastic model glider pilots who make their gliders perform fast circular flights near the top of a ridge subjected to a strong wind on one side and to nearly dead still air on the other side; an activity that led to an informal race of breaking speed records, the impressive one in date of April 13, 2017 being of $519 \mathrm{mph}(835 \mathrm{~km} / \mathrm{h})$. It is further boosted by the rapidly expanding market of drones and the mid-term perspective of motorized gliders that could fly autonomously over long distances by using DS in complementation of more classical energy harvesting techniques (solar charged batteries, thermal and slope upwind currents).

The present study revisits DS by exploiting a flight dynamics model previously used to work out nonlinear autopilots for scale-model airplanes [15-18]. A difference with models considered in other engineering-oriented DS studies is that this model does not explicitly involve a wind-frame, with associated attack and slide-slip angles, nor flight-path, bank, and heading angles commonly used in aircraft dynamics equations and for control design. These angle representations present singularities and add, from experience, useless complexities to flight control design and analysis. The approach here adopted also departs from other studies on DS, which focus on the characterization of trajectories that are optimal in terms of energy use. Search of optimality is here left aside for the development of new means of computer simulation allowing for easy testing of any glider traveling along any (mathematically specified) trajectory and confronted to any (mathematically specified) 
wind profile. We believe that such a simulation facility, even though it is not perfect because it relies on a simplified model of aerodynamic forces acting on the vehicle, is useful to more thoroughly grasp the possibilities offered by DS.

The paper is organized as follows. Section II presents the point-mass flight dynamics model subsequently used to simulate and analyze the flight of a glider along a path that intersects a wind shear layer, and derives energy-related equations that enlightens the process of energy-harvesting associated with specific flight maneuvers. Section III is devoted to the study of DS along an inclined circular path, i.e. a model of what is commonly called a Rayleigh cycle, and to the estimation, based on approximations, of various flight and environmental variables associated with DS. For small inclinations of the circle with respect to (w.r.t.) the horizontal plane, some of these estimates, namely the maximum glider airspeed as a function of the wind speed, and the corresponding circle's radius, essentially coincide with estimates previously derived by Richardson [19]. These estimates are of interest because they account for the impressive performance obtained by pilots of radio-controlled (RC) gliders. The technical arguments on which Richardson's calculations rely are obviously related to those used here. Being more elaborate our approach further allows us to work out estimates of other physical variables (speed increase after each cycle and minimal wind speed for sustained DS flight, in particular) that have not, to our knowledge, been derived elsewhere. Although the nonlinear flight dynamics model equations cannot be integrated explicitly, it is proved in Section IV that the corresponding state equations, given a mathematical expression of the followed flightpath as a function of the curvilinear abscissa and a mathematical expression of the wind profile in $3 D$-space, can be written in the form of an ordinary differential equation (ODE); an equation that can in turn be numerically integrated using a standard numerical integration package. This possibility is much related to the model of aerodynamic forces here considered. Simulation of DS along three different paths are subsequently considered for illustration purposes. The first path is the inclined circle considered in the previous section. It also serves to compare simulated values with values calculated with the estimates derived in this section, and observe their good concordance over a large spectrum of operating conditions. The second path is an inclined Lissajous curve whose eight shape is reminiscent of albatrosses closed trajectories evoked in various studies (see, $[3,5]$, 


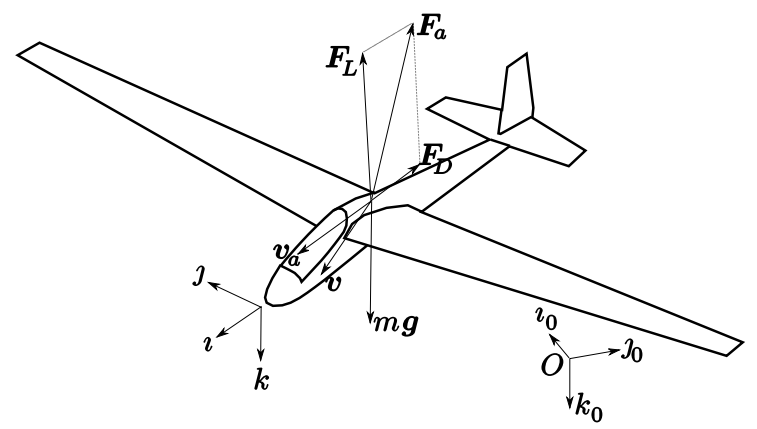

Fig. 1 Frames and forces

for instance). The third path is an inclined sinusoidal open curve, also reminiscent of observed albatrosses trajectories, for which simulated sustained flight results are reported for different angles between the wind direction and the overall path direction. Finally, a short summary of the original contributions presented in this paper is given in the concluding Section V.

\section{Modeling issues}

\section{A. Aerodynamic forces}

The resultant aerodynamic force $\boldsymbol{F}_{a}$ applied to a rigid body moving with air-velocity $\boldsymbol{v}_{a}$ is traditionally decomposed into the sum of a $d r a g$ force $\boldsymbol{F}_{D}$ along the direction of $\boldsymbol{v}_{a}$ and a lift force $\boldsymbol{F}_{L}$ perpendicular to this direction, i.e.

$$
\boldsymbol{F}_{a}=\boldsymbol{F}_{D}+\boldsymbol{F}_{L}
$$

The intensities of drag and lift forces are essentially proportional to $\left|\boldsymbol{v}_{a}\right|^{2}$ modulo variations characterized by two dimensionless functions $C_{D}$ and $C_{L}$, which depend in the first place on the orientation of $\boldsymbol{v}_{a}$ w.r.t. the body, but also on the Reynolds number $R_{e}$ and Mach number $M$. These dimensionless functions are called the aerodynamic characteristics of the body, or drag coefficient and lift coefficient respectively. More precisely

$$
\boldsymbol{F}_{D}=-\eta_{a}\left|v_{a}\right| C_{D} \boldsymbol{v}_{a}, \quad \boldsymbol{F}_{L}=\eta_{a}\left|v_{a}\right| C_{L} \boldsymbol{v}_{a}^{\perp}
$$

with $\boldsymbol{v}_{a}^{\perp}$ denoting some vector perpendicular to $\boldsymbol{v}_{a}$ and such that $\left|\boldsymbol{v}_{a}^{\perp}\right|=\left|v_{a}\right|$, and $\eta_{a}:=\frac{\rho \Sigma}{2}$ with $\rho$ the free stream air density, and $\Sigma$ an area germane to the body shape. 
It is well known that the norm of aerodynamic forces are commensurate with the squared norm of the air velocity so that one can safely assume that there exists two positive numbers $c$ and $d$ such that

$$
\left|\boldsymbol{F}_{a}\right|<c+d\left|\boldsymbol{v}_{a}\right|^{2}
$$

Let us define $\imath$ as the unit vector in the zero-lift direction of the aircraft, i.e. the direction of the air velocity perpendicular to the main wing's axis for which no lift force is produced, and $k$ the unit vector perpendicular to $\imath$ and to the main wing's axis (see Fig. 1). With this choice of the body frame the direction of $\boldsymbol{v}_{a}$ can be characterized by two angles $\alpha$ and $\beta$ such that $\boldsymbol{v}_{a}=$ $\left|\boldsymbol{v}_{a}\right|(\cos \alpha(\cos \beta \boldsymbol{\imath}+\sin \beta \boldsymbol{\jmath})+\sin \alpha \boldsymbol{k})$ with $\alpha=\arcsin \left(v_{a, 3} /\left|\boldsymbol{v}_{a}\right|\right)$ and $\beta=\arctan \left(v_{a, 2} / v_{a, 1}\right)$ denoting the angle of attack (here chosen equal to zero when the air velocity direction coincides with the aircraft zero-lift direction) and side-slip angle respectively. For this study of wind soaring we propose to work with the model of aerodynamic forces previously used in [17] for the design of scale-model aircraft autopilots. This model is:

$$
\boldsymbol{F}_{a}=-\left(c_{0} v_{a, 1} \boldsymbol{\imath}+\bar{c}_{0} v_{a, 3} \boldsymbol{k}\right)\left|\boldsymbol{v}_{a}\right|+v_{a, 2} \boldsymbol{O}\left(\boldsymbol{v}_{a}\right)
$$

with $c_{0}$ and $c_{1}$ denoting positive numbers, $\bar{c}_{0}=c_{0}+2 c_{1}$, and $\boldsymbol{O}\left(\boldsymbol{v}_{a}\right)$ any Euclidean vector-valued function such that the ratio $\frac{\left|\boldsymbol{O}\left(\boldsymbol{v}_{a}\right)\right|}{\left|\boldsymbol{v}_{a}\right|}$ is bounded. For instance, in [16] we have used $\boldsymbol{O}\left(\boldsymbol{v}_{a}\right)=-c_{0}\left|\boldsymbol{v}_{a}\right| \boldsymbol{J}$ for a model of a disc-shaped aircraft. A noticeable feature of this model is that it avoids the introduction of various angles, with the singularities associated with them, to parametrize the glider's attitude. In this respect it is sufficient to consider the rotation matrix associated with the body frame $\mathcal{B}$ and whose columns are formed by the vectors of coordinates of the unit Euclidean vectors $\boldsymbol{\imath}, \boldsymbol{\jmath}$ and $\boldsymbol{k}$. As required by physics, relation (4) is compatible with the assumed aircraft symmetry about the plane $(G ; \boldsymbol{r}, \boldsymbol{k})$. Note that if the drag coefficient $c_{0}$ were equal to zero then, in the case of zero side-slip angle (i.e. $v_{a, 2}=\beta=0$ ), the resultant aerodynamic force would be orthogonal to the zero-lift plane with an amplitude proportional to $\sin \alpha\left|\boldsymbol{v}_{a}\right|^{2}$. This model is also compatible with relations (1) and (2). Indeed, one verifies that, in this case, $\boldsymbol{v}_{a}^{\perp}=-\frac{\left|v_{a}\right|}{\cos \alpha} \boldsymbol{k}+\tan \alpha \boldsymbol{v}_{a}, C_{D}(\alpha)=\left(c_{0}+2 c_{1} \sin ^{2} \alpha\right) / \eta_{a}$, and $C_{L}(\alpha)=c_{1} \sin 2 \alpha / \eta_{a}$. For small angles of attack the drag coefficient $C_{D}$ is thus approximately equal to $\frac{c_{0}}{\eta_{a}}$ and the lift coefficient $C_{L}$ is approximately proportional to the angle of attack with the 
coefficient of proportionality given by $\frac{2 c_{1}}{\eta_{a}}$. This is coherent with conventional models of aerodynamic forces exerted on an aircraft, and with experimental data performed on a variety of wing profiles and axisymmetric bodies [20].

From now on we will assume that the glider is controlled so as to maintain a balanced flight, i.e. with no slide-slip or, equivalently, such that $v_{a, 2}=0$ [? ]. Under this assumption the resultant aerodynamic force (4) simplifies to

$$
\boldsymbol{F}_{a}=-\left(c_{0} v_{a, 1} \boldsymbol{\imath}+\bar{c}_{0} v_{a, 3} \boldsymbol{k}\right)\left|\boldsymbol{v}_{a}\right|
$$

Remark: This equation may, at first glance, look too simple to correctly model the aerodynamic forces acting on a glider. In fact, for small angles of attack, it closely matches other models used in the literature to model and analyze dynamic soaring. For instance, linear approximation about $\alpha=0$ of the lift and drag coefficients associated with this model yields the commonly used relation $C_{D}=C_{D_{0}}+k C_{L}^{2}[2-6,11]$ with, in this case, $C_{D_{0}}=c_{0} / \eta_{a}$ and $k=0.5 \eta_{a} / c_{1}$. Compared to classical models of lift that are linear or polynomial w.r.t. the angle of attack, the nonlinear model (5) also presents the advantage of complying with the physical property of zero lift when $\alpha= \pm \pi / 2$. This latter feature is useful to the design of controllers for scale-model aircraft which, due to their small size and inertia, are particularly sensitive to wind gusts and thus to large angle of attack excursions. For the present study of DS, a complementary interesting feature of this model is that it yields motion equations along pre-specified geometric paths that can be written in the form of easily (numerically) integrable ODEs. Using this property to simulate DS is detailed further on.

\section{B. Dynamic equations and energy considerations}

The aircraft dynamics related to the motion of the aircraft CoM are given by the classical Newton equation

$$
m \boldsymbol{a}=m \boldsymbol{g}+\boldsymbol{F}_{a}
$$

so that, assuming a balanced flight and in view of (5)

$$
m \boldsymbol{a}=m g_{0} \boldsymbol{k}_{0}-\left(c_{0}\left(\boldsymbol{v}_{a} \cdot \boldsymbol{\imath}\right) \boldsymbol{\imath}+\bar{c}_{0}\left(\boldsymbol{v}_{a} \cdot \boldsymbol{k}\right) \boldsymbol{k}\right)\left|\boldsymbol{v}_{a}\right|
$$


The dynamic equation (7) points out the importance of the coefficients $c_{0}$ and $\bar{c}_{0}$ to characterize the flight properties of the glider. In particular, the maximum glide rate of the aircraft, i.e. its glide ratio here denoted as $g r$, is a simple function of these coefficients. Indeed

$$
g r=\sup _{\alpha} \frac{\left|\boldsymbol{F}_{L}(\alpha)\right|}{\left|\boldsymbol{F}_{D}(\alpha)\right|}=\sup _{\alpha} \frac{\left|C_{L}(\alpha)\right|}{\left|C_{D}(\alpha)\right|}
$$

with

$$
\frac{C_{L}(\alpha)}{C_{D}(\alpha)}=\frac{2 c_{1}}{\bar{c}_{0} \tan (\alpha)+c_{0} \cot (\alpha)}
$$

whose derivative w.r.t the angle of attack is

$$
\frac{\partial}{\partial \alpha} \frac{C_{L}(\alpha)}{C_{D}(\alpha)}=-2 c_{1} \frac{\bar{c}_{0} / \cos ^{2}(\alpha)-c_{0} / \sin ^{2}(\alpha)}{\left(\bar{c}_{0} \tan (\alpha)+c_{0} \cot (\alpha)\right)^{2}}
$$

The largest value of $\left|C_{L}(\alpha)\right| /\left|C_{D}(\alpha)\right|$ is obtained by zeroing this derivative, i.e. for $\tan (\alpha)=\sqrt{\frac{c_{0}}{\bar{c}_{0}}}$. Using this latter relation, and $2 c_{1}=\bar{c}_{0}-c_{0}$, in (9) yields

$$
g r=\frac{\bar{c}_{0}-c_{0}}{2 \sqrt{c_{0} \bar{c}_{0}}}
$$

Because $c_{0}$ is typically much smaller than $\bar{c}_{0}$ in the case of fixed-wing aircraft, a good approximation of the previous expression of $g r$ is

$$
g r \approx 0.5 \sqrt{\bar{c}_{0} / c_{0}}
$$

As for the corresponding gliding speed $v_{g r}$, i.e. the aircraft speed along the optimal gliding path (in terms of the longest traveled distance before touching ground), using again $c_{0} \ll \bar{c}_{0}$ one finds that

$$
v_{g r} \approx \sqrt{m g_{0}} /\left(c_{0} \bar{c}_{0}\right)^{0.25}
$$

The corresponding sink rate is thus given by $v_{s i n k} \approx v_{g r} / g r \approx 2 \sqrt{m g_{0}} c_{0}^{0.25} / \bar{c}_{0}^{0.75}$. Conversely, $\bar{c}_{0}$ and $c_{0}$ can be calculated from $g r$ and $v_{g r}$ according to

$$
\bar{c}_{0} \approx \frac{2 g r}{v_{g r}^{2}} m g_{0}, \quad c_{0} \approx \frac{m g_{0}}{2 g r v_{g r}^{2}}
$$

Now, let $z$ denote the glider's altitude and $E:=0.5 m|v|^{2}+m g_{0} z$ the total (kinematic + potential) energy of the glider. Because $\dot{z}=-\boldsymbol{k}_{0} \cdot \boldsymbol{v}$, the inner product of both members of the equality (7) by $\boldsymbol{v}$ yields

$$
\dot{E}=-\left(c_{0}\left(\boldsymbol{v}_{a} \cdot \boldsymbol{\imath}\right)(\boldsymbol{v} \cdot \boldsymbol{\imath})+\bar{c}_{0}\left(\boldsymbol{v}_{a} \cdot \boldsymbol{k}\right)(\boldsymbol{v} \cdot \boldsymbol{k})\right)\left|\boldsymbol{v}_{a}\right|
$$


Sustained flight requires $E$ to be bounded from below. This in turn implies that losses of energy during some parts of the flight must be compensated by energy increases during other parts of the flight. It is well known that such increases can be obtained by taking advantage of ascending thermal currents, i.e. when $\boldsymbol{v}_{w}$ contains an upward vertical component. Dynamic soaring poses the question of whether energy increases can also be obtained when the wind blows horizontally during some parts of the flight. In this respect equation (14) is important to understand the energy production/dissipation process associated with dynamic soaring. First this equation shows that in the absence of wind, i.e. when $\boldsymbol{v}_{w}=0, \dot{E}=-\left(c_{0}(\boldsymbol{v} . \boldsymbol{\imath})^{2}+\bar{c}_{0}(\boldsymbol{v} \cdot \boldsymbol{k})^{2}\right)|\boldsymbol{v}|<0$. The total energy of the glider thus always decreases in this case -a physically coherent property implying that a glider maintaining its speed in still air constantly loses altitude. Then it shows that, when the angle of attack is small (which implies that $\left|\boldsymbol{v}_{a} . \boldsymbol{\imath}\right| \approx\left|\boldsymbol{v}_{a}\right|$ ) and $\left|\boldsymbol{v}_{w}\right|<|\boldsymbol{v}|$, the term $-c_{0}\left(\boldsymbol{v}_{a} . \boldsymbol{\imath}\right)(\boldsymbol{v} . \boldsymbol{\imath})\left|\boldsymbol{v}_{a}\right|$ is negative and thus dissipates energy. Nevertheless, because $c_{0} \ll \bar{c}_{0}$ in the case of airplanes and gliders, this term can be small compared to the second term $-\bar{c}_{0}\left(\boldsymbol{v}_{a} \cdot \boldsymbol{k}\right)(\boldsymbol{v} \cdot \boldsymbol{k})\left|\boldsymbol{v}_{a}\right|$ in the right-hand side of (14) so that, in the first approximation, the glider's energy varies according to

$$
\dot{E} \approx-\bar{c}_{0}\left(\boldsymbol{v}_{a} \cdot \boldsymbol{k}\right)(\boldsymbol{v} \cdot \boldsymbol{k})\left|\boldsymbol{v}_{a}\right|
$$

Because the angle of attack is nominally positive and smaller than $\pi / 2$, the scalar product $\boldsymbol{v}_{a} \cdot \boldsymbol{k}$ is positive. Equation (14), or (15), indicates that the total energy of the glider can increase only when $\boldsymbol{v}_{a} . \boldsymbol{k}$ and $\boldsymbol{v} . \boldsymbol{k}$ have opposite signs. Energy transfer issues are pointed out and discussed in all DS studies, starting with [1] and, more recently, $[4,5,7]$. All explain that energy is gained when there is wind and the glider performs specific maneuvers in relation to the wind's direction. However, to our knowledge, the condition of energy increase has not previously been mathematically characterized by the simple geometrical inequality $\left(\boldsymbol{v}_{a} \cdot \boldsymbol{k}\right)(\boldsymbol{v} \cdot \boldsymbol{k})<0$. A typical maneuver, evoked in most DS studies, for which this inequality is satisfied is a circular (quasi-horizontal) half-turn started by facing the horizontal wind, and performed with enough speed so that the glider has to lean strongly into the turn. Its main wing then tends to become vertical and uses the wind much alike the propulsive sail of a sailing boat. Figure 2 shows a schematic representation of the projections on the circle's plane of the involved velocities and of the unit vectors $\boldsymbol{\imath}$ and $\boldsymbol{k}$ during such a maneuver. The part of the circle where energy can be harvested from the wind, i.e. where $\boldsymbol{v} . \boldsymbol{k}<0$ is colored in blue. However, 


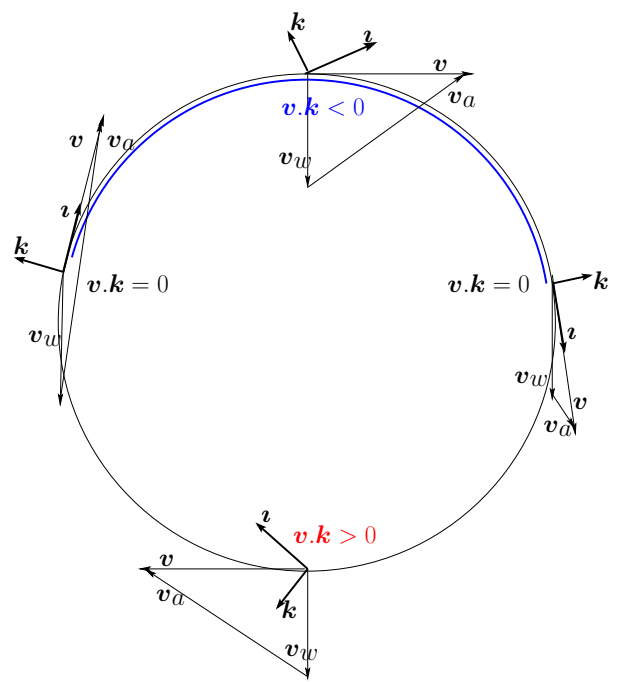

Fig. 2 Variations of $v . k$ along a circular horizontal path

$\boldsymbol{v} \boldsymbol{k}$ is positive when the glider follows the other part of the circle with the same horizontal wind, and the energy balance over the whole circle is negative, implying that sustained level flight is then not possible. As a matter of fact this balance turns out to be worse than in the no-wind case. The question then becomes to find out whether a reduction of the wind speed on this second part can reduce the energy loss so that the balance can be reached over the complete cycle. In other words can horizontal wind gradients be used to achieve a sustained flight? This question brings us back to studying Rayleigh's cycles evoked in all works on dynamic soaring, not only for theoretical reasons but also to tentatively account for the impressive performances recently obtained by model glider pilots.

\section{Rayleigh's cycle and estimation of dynamic soaring characteristics}

A Rayleigh cycle, first envisioned by Lord Rayleigh [1] who was interested in the flight of large birds without working their wings like the albatross, is sketched on Figure 3. The principle is as follows: the glider descends downwind along a circular-like path, passes through a horizontal shear boundary into a layer of slower or stationary air, turns upwind, passes again through the shear boundary to face the wind, and so on. Alike many authors we will here model the path followed by the glider by a circle of radius $r$ inclined with an angle $\theta(\neq 0)$ w.r.t the horizontal plane (see 


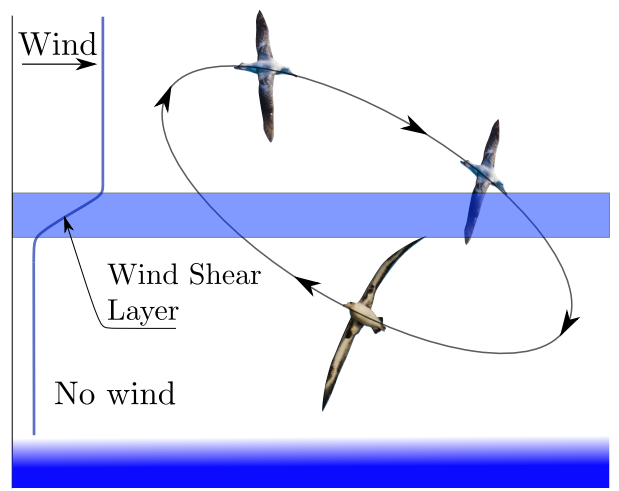

Fig. 3 Rayleigh cycle

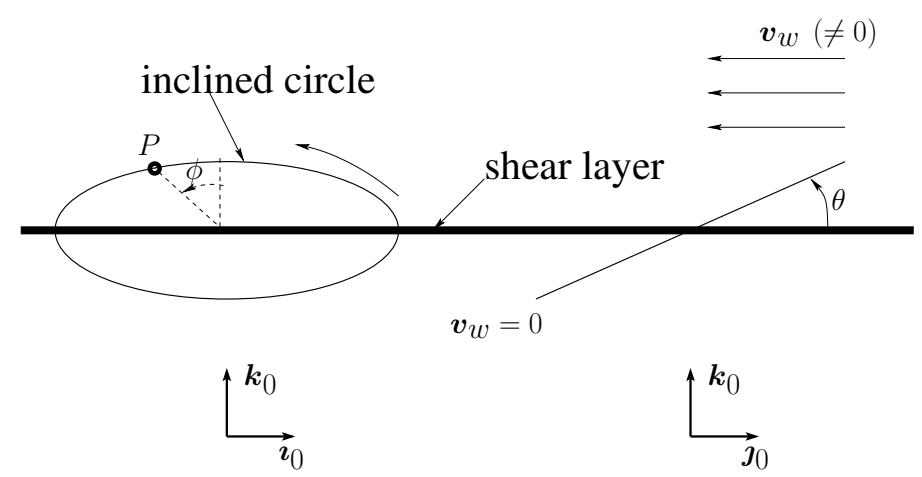

Front view

Side View

Fig. 4 Circle, wind, and inertial frame vectors

Figure 4). The shear layer is supposed to be "thin" and it crosses the circle along a diameter. The wind velocity $\boldsymbol{v}_{w}$ above the shear layer is supposed constant and perpendicular to this diameter. The corresponding wind speed, equal to $\left|\boldsymbol{v}_{w}\right|$, is denoted as $v_{w}$ to lighten the notation. Below the shear layer the air is still. The wind's gradient w.r.t. the altitude between the shear boundaries can be modeled by any smooth (say twice differentiable) monotonic function.

Because $\dot{\boldsymbol{v}}_{a}=\boldsymbol{a}-\dot{\boldsymbol{v}}_{w}$ one deduces from (7) that

$$
m \dot{\boldsymbol{v}}_{a}=-m g_{0} \boldsymbol{k}_{0}-\left(c_{0}\left(\boldsymbol{v}_{a} \cdot \boldsymbol{\imath}\right) \boldsymbol{\imath}+\bar{c}_{0}\left(\boldsymbol{v}_{a} \cdot \boldsymbol{k}\right) \boldsymbol{k}\right)\left|\boldsymbol{v}_{a}\right|-m \dot{\boldsymbol{v}}_{w}
$$

Using the facts that $\boldsymbol{k}_{0} \cdot \boldsymbol{v}_{w}=0$, because the wind blows horizontally by assumption, and $\left|\boldsymbol{v}_{a}\right|^{2}=$ $\left(\boldsymbol{v}_{a} \cdot \boldsymbol{\imath}\right)^{2}+\left(\boldsymbol{v}_{a} \cdot \boldsymbol{k}\right)^{2}$, because $v_{a, 2}=0$ by assumption of a balanced flight, the scalar product of both 
members of the equality (16) with $\boldsymbol{v}_{a}$ yields

$$
\begin{aligned}
m \dot{\boldsymbol{v}}_{a} \cdot \boldsymbol{v}_{a}= & -m g_{0}\left(\boldsymbol{k}_{0} \cdot \boldsymbol{v}\right)-\left(c_{0}\left(\boldsymbol{v}_{a} \cdot \boldsymbol{\imath}\right)^{2}+\bar{c}_{0}\left(\boldsymbol{v}_{a} \cdot \boldsymbol{k}\right)^{2}\right)\left|\boldsymbol{v}_{a}\right| \\
& -m \dot{\boldsymbol{v}}_{w} \cdot \boldsymbol{v}_{a} \\
= & -m g_{0}\left(\boldsymbol{k}_{0} \cdot \boldsymbol{v}\right)-c_{0}\left|\boldsymbol{v}_{a}\right|^{3}-2 c_{1}\left(\boldsymbol{v}_{a} \cdot \boldsymbol{k}\right)^{2}\left|\boldsymbol{v}_{a}\right| \\
& +m \dot{\boldsymbol{v}}_{w} \cdot \boldsymbol{v}_{w}-m \dot{\boldsymbol{v}}_{w} \cdot \boldsymbol{v}
\end{aligned}
$$

Define the auxiliary energy function $\bar{E}:=E_{a}-0.5 m\left|\boldsymbol{v}_{w}\right|^{2}$, with $E_{a}:=0.5 m\left|\boldsymbol{v}_{a}\right|^{2}+m g_{0} z$ denoting the glider's total energy w.r.t. the moving ambient air. Note that $\bar{E}=E$ when the glider's inertial velocity is perpendicular to the wind direction. The equality (17) may also be written as

$$
\dot{\bar{E}}=-c_{0}\left|\boldsymbol{v}_{a}\right|^{3}-2 c_{1}\left(\boldsymbol{v}_{a} \cdot \boldsymbol{k}\right)^{2}\left|\boldsymbol{v}_{a}\right|-m \dot{\boldsymbol{v}}_{w} \cdot \boldsymbol{v}
$$

Let us now evaluate the modification of $\bar{E}$ or, equivalently, of $E$ on a cycle, i.e. between a time-instant $t=0$ when the glider leaves the top of the circle and the time-instant $T$ when it returns for the first time to this position. From the definition of $\bar{E}$ and using the equalities $\boldsymbol{v}(0) \cdot \boldsymbol{v}_{w}(0)=\boldsymbol{v}(T) \cdot \boldsymbol{v}_{w}(T)=0$ so that $\left|\boldsymbol{v}_{a}(T)\right|^{2}-\left|\boldsymbol{v}_{a}(0)\right|^{2}=|\boldsymbol{v}(T)|^{2}-|\boldsymbol{v}(0)|^{2}$, and $z(0)=z(T)$, the integration of (18) on the time interval $[0, T]$ yields

$$
\begin{aligned}
\bar{E}(T)-\bar{E}(0)= & 0.5 m\left(|\boldsymbol{v}(T)|^{2}-|\boldsymbol{v}(0)|^{2}\right) \\
= & \int_{t=0}^{T}-c_{0}\left|\boldsymbol{v}_{a}(s)\right|^{3} d s \\
& +\int_{t=0}^{T}-2 c_{1}\left(\boldsymbol{v}_{a}(s) \cdot \boldsymbol{k}(s)\right)^{2}\left|\boldsymbol{v}_{a}(s)\right| d s \\
& +\int_{t=0}^{T}-m \dot{\boldsymbol{v}}_{w}(s) \cdot \boldsymbol{v}(s) d s
\end{aligned}
$$

Note that the first two integrals in the right-hand side of this equality are negative. They are energy dissipative terms. Therefore, the third integral is the only one that can increase the energy and yield a sustained flight. The explicit calculation of these integrals is not possible. Instead, we propose to estimate them by using approximations that are best justified when the difference between $|\boldsymbol{v}|$ and $\left|\boldsymbol{v}_{a}\right|$, and the relative variations of $|\boldsymbol{v}|$ on the circle, are small. To this purpose we denote the glider's average speed on the circle as $\bar{v}$ so that $T \approx \frac{2 \pi r}{\bar{v}}$ and

$$
\begin{aligned}
\int_{t=0}^{T}-c_{0}\left|\boldsymbol{v}_{a}(s)\right|^{3} d s & \approx-c_{0} T \bar{v}^{3} \\
& \approx-2 \pi r c_{0} \bar{v}^{2}
\end{aligned}
$$

Concerning the third integral, let $v_{\text {down }}\left(\right.$ resp. $v_{u p}$ ) denote the glider speed when it crosses the boundary layer going down (resp. going up). Let also $t_{1}$ (resp. $\left.t_{1}+\delta_{1}\right)$ and $t_{2}$ (resp. $t_{2}+\delta_{2}$ ) denote 
the time-instants when the glider enters (resp. leaves) the shear layer. Because the shear layer is thin we may assume that the glider speed is almost unchanged when crossing it. Therefore

$$
\begin{aligned}
& \boldsymbol{v}(s) \approx \begin{cases}v_{\text {down }}\left(-\cos (\theta) \boldsymbol{j}_{0}-\sin (\theta) \boldsymbol{k}_{0}\right), & s \in\left[t_{1}, t_{1}+\delta_{1}\right] \\
v_{u p}\left(\cos (\theta) \boldsymbol{j}_{0}+\sin (\theta) \boldsymbol{k}_{0}\right), & s \in\left[t_{2}, t_{2}+\delta_{2}\right]\end{cases} \\
& \boldsymbol{v}_{w}\left(t_{1}\right)=-v_{w} \boldsymbol{J}_{0}, \boldsymbol{v}_{w}\left(t_{1}+\delta_{1}\right)=0 \\
& \boldsymbol{v}_{w}\left(t_{2}\right)=0, \quad \boldsymbol{v}_{w}\left(t_{2}+\delta_{2}\right)=-v_{w} \boldsymbol{J}_{0}
\end{aligned}
$$

By further assuming that $|\boldsymbol{v}|$ is approximately constant (and approximately equal to the average velocity $\bar{v}$ ) on the upper part of the circle, using the circle's symmetry yields

$$
\begin{aligned}
\int_{t=0}^{T}-m \dot{\boldsymbol{v}}_{w}(s) \cdot \boldsymbol{v}(s) d s \approx & -m\left(\boldsymbol{v}\left(t_{1}\right) \cdot \int_{t_{1}}^{t 1+\delta_{1}} \dot{\boldsymbol{v}}_{w}(s) d s\right. \\
& \left.+\boldsymbol{v}\left(t_{2}\right) \cdot \int_{t_{2}}^{t 2+\delta_{2}} \dot{\boldsymbol{v}}_{w}(s) d s\right) \\
= & m\left(v_{d o w n}+v_{u p}\right) v_{w} \cos (\theta) \\
\approx & 2 m \bar{v} v_{w} \cos (\theta)
\end{aligned}
$$

This relation shows that the balance of kinetic energy increment due to crossing the shear layer is approximately proportional to the wind speed above the shear layer, and decreases with the angle of inclination of the circular path w.r.t. the horizontal plane.

Let us now estimate the second integral. By assuming that the glider speed is approximately equal to the average speed $\bar{v}$, the glider's acceleration $\boldsymbol{a}$ is approximately equal to $\frac{\bar{v}^{2}}{r} \overline{\boldsymbol{u}}$ with $\overline{\boldsymbol{u}}$ denoting the unit vector pointing from the glider's CoM to the circle's center. Using this approximation in the dynamic equation (7) yields

$$
m \frac{\bar{v}^{2}}{r} \overline{\boldsymbol{u}}-m g_{0} \boldsymbol{k}_{0} \approx-\left(c_{0}\left(\boldsymbol{v}_{a} \cdot \boldsymbol{\imath}\right) \boldsymbol{\imath}+\bar{c}_{0}\left(\boldsymbol{v}_{a} \cdot \boldsymbol{k}\right) \boldsymbol{k}\right)\left|\boldsymbol{v}_{a}\right|
$$

Forming the squared norm of both members of this (near) equality then yields

$$
m^{2}\left(\frac{\bar{v}^{4}}{r^{2}}+g_{0}^{2}-2 g_{0} \frac{\bar{v}^{2}}{r}\left(\overline{\boldsymbol{u}} \cdot \boldsymbol{k}_{0}\right)\right) \approx\left(c_{0}^{2}\left(\boldsymbol{v}_{a} \cdot \boldsymbol{\imath}\right)^{2}+\bar{c}_{0}^{2}\left(\boldsymbol{v}_{a} \cdot \boldsymbol{k}\right)^{2}\right)\left|\boldsymbol{v}_{a}\right|^{2}
$$

Because $\left|\boldsymbol{v}_{a}\right|^{2}=\left(\boldsymbol{v}_{a} \cdot \boldsymbol{\imath}\right)^{2}+\left(\boldsymbol{v}_{a} \cdot \boldsymbol{k}\right)^{2}$, we have also

$$
m^{2}\left(\frac{\bar{v}^{4}}{r^{2}}+g_{0}^{2}-2 g_{0} \frac{\bar{v}^{2}}{r}\left(\overline{\boldsymbol{u}} \cdot \boldsymbol{k}_{0}\right)\right) \approx c_{0}^{2}\left|\boldsymbol{v}_{a}\right|^{4}+\left(\bar{c}_{0}^{2}-c_{0}^{2}\right)\left(\boldsymbol{v}_{a} \cdot \boldsymbol{k}\right)^{2}\left|\boldsymbol{v}_{a}\right|^{2}
$$

Using again the approximation $\left|\boldsymbol{v}_{a}\right| \approx \bar{v}$, and because $c_{0} \ll \bar{c}_{0}$, the previous relation yields (recall also that $\bar{c}_{0}=2 c_{1}+c_{0} \approx 2 c_{1}$ )

$$
2 c_{1}\left(\boldsymbol{v}_{a} \cdot \boldsymbol{k}\right)^{2}\left|\boldsymbol{v}_{a}\right| \approx \frac{1}{\bar{c}_{0}}\left(\frac{m^{2}}{r^{2}}-c_{0}^{2}\right) \bar{v}^{3}+\frac{m^{2} g_{0}^{2}}{\bar{c}_{0} \bar{v}}-\frac{2 m^{2} g_{0} \bar{v}}{\bar{c}_{0} r}\left(\overline{\boldsymbol{u}} \cdot \boldsymbol{k}_{0}\right)
$$


Assuming a near constant speed, the integration of $\left(\overline{\boldsymbol{u}} \cdot \boldsymbol{k}_{0}\right)$ on the circle vanishes. Integration of both members of the previous (near) equality, with $T \approx \frac{2 \pi r}{\bar{v}}$, yields

$$
\begin{aligned}
\int_{t=0}^{T}-2 c_{1}\left(\boldsymbol{v}_{a}(s) \cdot \boldsymbol{k}(s)\right)^{2}\left|\boldsymbol{v}_{a}(s)\right| d s \approx & -\frac{2 \pi}{\bar{c}_{0}}\left(\frac{m^{2}}{r}-r c_{0}^{2}\right) \bar{v}^{2} \\
& -\frac{2 \pi r m^{2} g_{0}^{2}}{\bar{c}_{0} \bar{v}^{2}}
\end{aligned}
$$

Using (20)-(22) in (19) with $c_{0} \ll \bar{c}_{0}$, and using also the fact that $c_{0} \ll 1$ in the case of airplanes and gliders so that $c_{0}^{2} \ll c_{0}$, then yields the following approximation

$$
|\boldsymbol{v}(T)|^{2}-|\boldsymbol{v}(0)|^{2} \approx 4\left(\cos (\theta) v_{w} \bar{v}-\pi\left(\frac{m}{\bar{c}_{0} r}+\frac{c_{0} r}{m}\right) \bar{v}^{2}-\pi \frac{m g_{0}^{2} r}{\bar{c}_{0} \bar{v}^{2}}\right)
$$

Let $\Delta v:=|\boldsymbol{v}(T)|-|\boldsymbol{v}(0)|$ denote the change of speed after the glider has completed one circle, so that $|\boldsymbol{v}(T)|^{2}-|\boldsymbol{v}(0)|^{2} \approx 2 \bar{v} \Delta v$. Then, in view of (23) an estimation of $\Delta v$ is

$$
\Delta v \approx 2\left(\cos (\theta) v_{w}-\pi\left(\frac{m}{\bar{c}_{0} r}+\frac{c_{0} r}{m}\right) \bar{v}-\pi \frac{m g_{0}^{2} r}{\bar{c}_{0} \bar{v}^{3}}\right)
$$

To our knowledge such an expression of the speed variation over a Rayleigh cycle has not been derived before. Let $\bar{v}_{k}$ denote the glider's average speed on the time period $\left[T_{k}, T_{k+1}=T_{k}+\frac{2 \pi r}{\bar{v}_{k}}\right)$ needed to travel one complete circle. The previous relation suggests that $v_{k}$ evolves approximately according to

$$
\bar{v}_{k+1}=\bar{v}_{k}+2\left(\cos (\theta) v_{w}-\pi\left(\frac{m}{\bar{c}_{0} r}+\frac{c_{0} r}{m}\right) \bar{v}_{k}-\pi \frac{m g_{0}^{2} r}{\bar{c}_{0} \bar{v}_{k}^{3}}\right)
$$

Figure 5 shows this evolution in the case where the parameters of Table 1 (given in the international system of units (SI)) are used.

Table 1 A set of parameters

\begin{tabular}{ll}
\hline \hline glider & $m=3 \mathrm{~kg}, \mathrm{c}_{0}=0.001 \mathrm{~kg} / \mathrm{m}, \mathrm{c}_{1}=2 \mathrm{~kg} / \mathrm{m}$ \\
\hline circle & $r=50 \mathrm{~m}, \theta=0.2 \mathrm{rad}$ \\
\hline wind speed in upper layer & $v_{w}=10 \mathrm{~m} / \mathrm{s}$ \\
\hline \hline
\end{tabular}

The corresponding glide rate and gliding speed of the modeled glider, calculated from (11) and (12), are $g r=31.6$ and $v_{g r}=21.6 \mathrm{~m} / \mathrm{s}$.

Relation (24), taken as an equality, can in turn be used to estimate various dynamic soaring characteristics on a Rayleigh's circle, namely the minimal wind speed $v_{w, \text { min }}$ for a given circle's 


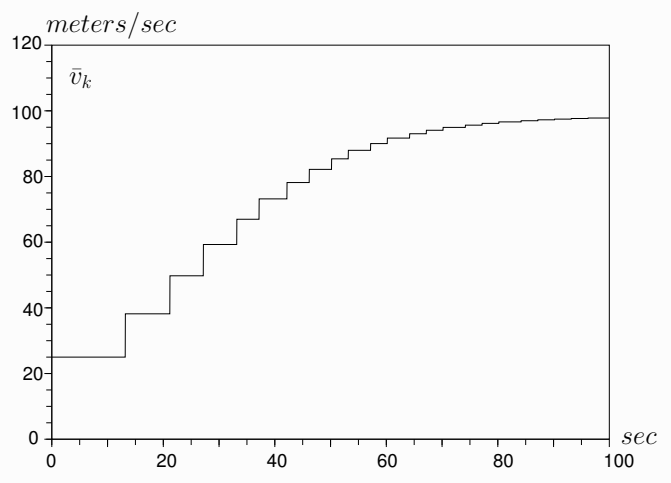

Fig. 5 Time-evolution of the estimated average speed

radius and below which sustained flight is not possible, the optimal radius $r_{o p t}$ yielding the highest possible speed for a given wind velocity, and the corresponding maximum speed $\bar{v}_{\max }$.

Estimation of $v_{w, m i n}$ : Dynamic soaring on a Rayleigh cycle is possible if there exists a speed range for which $\Delta v$ is positive or equal to zero. Therefore the maximum of $\Delta v$ w.r.t. $\bar{v}$ must be positive or equal to zero. From (24) one has

$$
\frac{\partial}{\partial \bar{v}} \Delta v=2 \pi\left(-\left(\frac{m}{\bar{c}_{0} r}+\frac{c_{0} r}{m}\right)+\frac{3 m g_{0}^{2} r}{\bar{c}_{0} \bar{v}^{4}}\right)
$$

and the maximum of $\Delta v$ is obtained when $\frac{\partial}{\partial \bar{v}} \Delta v=0$, i.e. for $\bar{v}=\bar{v}_{\text {min }}$ with

$$
\bar{v}_{\min }=\left(\frac{3 m^{2} g_{0}^{2}}{\left(\frac{m^{2}}{r^{2}}+c_{0} \bar{c}_{0}\right)}\right)^{0.25}
$$

This is also the (estimated) minimal average speed of a sustained flight. In the case of the parameters of Table 1 it is equal to $24 \mathrm{~m} / \mathrm{s}$. Using the previous relation in (24) yields

$$
\Delta v_{\max } \frac{\bar{v}^{3}}{2}=\cos (\theta) v_{w}\left(\frac{3 m^{2} g_{0}^{2}}{\left(\frac{m^{2}}{r^{2}}+c_{0} \bar{c}_{0}\right)}\right)^{0.75}-\frac{4 \pi m g_{0}^{2} r}{\bar{c}_{0}}
$$

so that $\Delta v_{\max }$ can be positive or equal to zero only if $v_{w} \geq v_{w, \min } / \cos (\theta)$ with

$$
v_{w, \min }=\frac{4 \pi r}{3^{0.75} \bar{c}_{0}}\left(\frac{g_{0}}{m}\right)^{0.5}\left(\frac{m^{2}}{r^{2}}+c_{0} \bar{c}_{0}\right)^{0.75}
$$

With the parameters of Table 1 this expression yields $v_{w, \min }=3.21 \mathrm{~m} / \mathrm{s}$. This approximation of the minimal wind speed, although slightly optimistic as we will later verify via simulation, is nonetheless of interest because it points out that sustained flight does not necessarily require a strong wind. 
Estimation of $\bar{v}_{\text {max }}$ : This estimate is obtained by zeroing $\Delta v$ and it is the finite limit -when it exists- of the sequence $\bar{v}_{k}(k \in \mathbb{N})$ of relation (25). This limit is easily computed numerically. It is also the largest real solution to the fourth-degree polynomial equation in $x$

$$
\cos (\theta) v_{w} x^{3}-\pi\left(\frac{m}{\bar{c}_{0} r}+\frac{c_{0} r}{m}\right) x^{4}-\pi \frac{m g_{0}^{2} r}{\bar{c}_{0}}=0
$$

In order to propose a simple explicit expression we propose an estimation obtained by assuming that the constant term in the left-hand side of this equality is dominated by the other two terms when the average soaring speed $\bar{v}$ is large. This yields the following estimate of $\bar{v}_{\max }$

$$
\bar{v}_{\text {max }} \approx \cos (\theta) \frac{v_{w}}{\frac{\pi r}{\bar{c}_{0} m}\left(\frac{m^{2}}{r^{2}}+c_{0} \bar{c}_{0}\right)}
$$

whose accuracy should thus increase with the size of this estimate.

Estimation of $r_{\text {opt }}$ : The optimization of the circle's radius depends on the chosen criterion. An option is to work out the radius for which sustained flight is possible with the smallest minimal wind speed $v_{w, \text { min }}$ given by (27). The corresponding solution is $r=\frac{m}{\sqrt{2 c_{0} \bar{c}_{0}}}$. The other option, here retained, is the radius yielding the fastest soaring speed, i.e. for which $\bar{v}_{\text {max }}$ is the largest. In view of (28) this is the value of $r$ that minimizes $r\left(\frac{m^{2}}{r^{2}}+c_{0} \bar{c}_{0}\right)$, i.e.

$$
r_{o p t} \approx \frac{m}{\sqrt{c_{0} \bar{c}_{0}}}=\frac{v_{g r}^{2}}{g_{0}}
$$

with $v_{g r}$ the gliding speed given by (12). This radius is equal to $47.4 m$ in the case of the glider parameters of Table 1. The corresponding soaring speed is

$$
\bar{v}_{\text {max } \mid r_{o p t}} \approx \cos (\theta) \frac{v_{w}}{2 \pi} \sqrt{\frac{\bar{c}_{0}}{c_{0}}}=\cos (\theta) \frac{v_{w}}{\pi} g r
$$

with $g r$ the glide rate given by (11), and the corresponding loop period is

$$
T_{o p t} \approx \frac{2 \pi r_{o p t}}{\bar{v}_{\text {max } \mid r_{o p t}}}=\frac{2 \pi v_{g r}^{2}}{g_{0} \bar{v}_{\text {max } \mid r_{o p t}}}
$$

Numerical values obtained with the glider parameters of Table 1 are $\bar{v}_{\text {max } \mid r_{\text {opt }}} \approx 98.7 \mathrm{~m} / \mathrm{s}$ and $T_{\text {opt }} \approx 3 s$. At this point it is worth noting that, in the particular case where the angle $\theta$ is very small so that $\cos (\theta)$ can be approximated by one, these last two estimates coincide with those proposed by Richardson [19] on the basis of a simpler model of the glider's dynamics. We anticipated this finding 
which points out the compatibility of our respective approaches. Ours, being more elaborate, goes with complementary predictions and a way of testing their accuracy via simulation.

Precise numerical integration of the glider's dynamic equations on the assigned path, comparison of observed simulation results with the estimates derived previously, and simulation of other operating conditions are addressed in the next Section.

\section{Simulation}

\section{A. Numerical integration of dynamic equations on a given arbitrary path}

Consider a regular (at least twice differentiable) $3 D$-path $\mathcal{C}$ parametrized by its curvilinear abscissa $s$ and a running point $P(s)$ on this curve whose Cartesian coordinates $(x, y, z)$ w.r.t. to the chosen inertial frame $\mathcal{I}=\left\{O ; \boldsymbol{\imath}_{0}, \boldsymbol{\jmath}_{0}, \boldsymbol{k}_{0}\right\}$ are specified either explicitly in terms of known functions of $s$, or via the point $P(0)$ complemented with coordinate-derivatives w.r.t. the curvilinear abscissa, i.e. $x^{\prime}(s)=f_{x}(x(s), y(s), z(s)), y^{\prime}(s)=f_{y}(x(s), y(s), z(s)), z^{\prime}(s)=f_{z}(x(s), y(s), z(s))$. In this latter case the functions $f_{x}, f_{y}$ and $f_{z}$ are given and such that $f_{x}^{2}+f_{y}^{2}+f_{z}^{2}=1$ (normalization constraint). The main issue then, given the initial position of $P$ on the path, is to determine the curvilinear abscissa at all time-instants, i.e. to numerically compute $s(t), \forall t \geq 0$.

Let $\boldsymbol{u}(s)$ denote the unit vector tangent to the path at the point $P(s)$, i.e.

$$
\boldsymbol{u}(s)=x^{\prime}(s) \boldsymbol{\imath}_{0}+y^{\prime}(s) \boldsymbol{\jmath}_{0}+z^{\prime}(s) \boldsymbol{k}_{0}
$$

When the point $P$ moves on the path, the time derivative of $\boldsymbol{u}$ is thus given by

$$
\dot{\boldsymbol{u}}(s, \dot{s})=\left(x^{\prime \prime}(s) \boldsymbol{\imath}_{0}+y^{\prime \prime}(s) \boldsymbol{\jmath}_{0}+z^{\prime \prime}(s) \boldsymbol{k}_{0}\right) \dot{s}
$$

The velocity of $P$ w.r.t. the inertial frame is the vector $\boldsymbol{v}=\dot{\boldsymbol{s}} \boldsymbol{u}$. Now, define the state vector $X:=(x, y, z, s, \dot{s})^{\top}$ and assume that the function $g_{s}(X, t)$ such that $\ddot{s}=g_{s}(X, t)$ is known to us, then the position of $P$ and its velocity at any time-instant can be calculated by numerically 
integrating the ordinary differential equation (ODE)

$$
\dot{X}=\left(\begin{array}{ccccc}
0 & 0 & 0 & 0 & x^{\prime}(s) \\
0 & 0 & 0 & 0 & y^{\prime}(s) \\
0 & 0 & 0 & 0 & z^{\prime}(s) \\
0 & 0 & 0 & 0 & 1 \\
0 & 0 & 0 & 0 & 0
\end{array}\right) X+\left(\begin{array}{c}
0 \\
0 \\
0 \\
0 \\
g_{s}(X, t)
\end{array}\right)
$$

from the initial condition $X(0)=\left(x_{0}, y_{0}, z_{0}, 0,\left|\dot{\boldsymbol{v}}_{0}\right|\right)^{\top}$. Using a standard numerical integration package, simulation of flight time-periods of several minutes then just takes a few seconds on an average PC.

Remark: In the case where the coordinates of $P$ are specified in terms of known functions of the curvilinear abscissa, it suffices to define the two-dimensional state $X:=(s, \dot{s})^{\top}$ and numerically integrate the corresponding ODE from the initial condition $X(0):=\left(0,\left|\dot{\boldsymbol{v}}_{0}\right|\right)^{\top}$.

In the context of dynamic soaring the point $P$ is the glider's CoM, and $\boldsymbol{v}$ is the glider's velocity on the chosen path $\mathcal{C}$. We show next that, given any continuous wind velocity function $\boldsymbol{v}_{w}(x, y, z, t)$, the function $g_{s}$ can be explicitly determined from the dynamic equation (7).

Determination of the function $g_{s}$ :

Because $\boldsymbol{v}=\dot{s} \boldsymbol{u}$, one deduces that

$$
\begin{aligned}
\boldsymbol{a} & =\ddot{\boldsymbol{u}} \boldsymbol{u}+\dot{\boldsymbol{s}} \boldsymbol{u} \\
& =\ddot{\boldsymbol{s}} \boldsymbol{u}+\dot{s}^{2} \boldsymbol{h}
\end{aligned}
$$

with $\boldsymbol{h}(s):=\left(x^{\prime \prime}(s) \boldsymbol{\imath}_{0}+y^{\prime \prime}(s) \boldsymbol{\jmath}_{0}+z^{\prime \prime}(s) \boldsymbol{k}_{0}\right)$. Using the fact that $\dot{\boldsymbol{u}}$, and thus $\boldsymbol{h}$, are orthogonal to $\boldsymbol{u}$ (because $\boldsymbol{u}$ is a unit vector), one deduces that

$$
|\boldsymbol{a}|^{2}=|\ddot{s}|^{2}+\dot{s}^{4}|\boldsymbol{h}|^{2}
$$

Define

$$
\overline{\boldsymbol{g}}:=\boldsymbol{g}-\frac{\bar{c}_{0}}{m} \boldsymbol{v}_{a}\left|\boldsymbol{v}_{a}\right|
$$

with $\boldsymbol{v}_{a}(X, t)=\dot{s} \boldsymbol{u}(s)-\boldsymbol{v}_{w}(x, y, z, t)$. Because $\boldsymbol{v}_{a}=\left(\boldsymbol{v}_{a} \cdot \boldsymbol{\imath}\right) \boldsymbol{\imath}+\left(\boldsymbol{v}_{a} \cdot \boldsymbol{k}\right) \boldsymbol{k}$ for a balanced flight, the 
dynamic equation (7) may also be written as

$$
\boldsymbol{a}-\overline{\boldsymbol{g}}=2 \frac{c_{1}}{m}\left|\boldsymbol{v}_{a}\right|\left(\boldsymbol{v}_{a} . \boldsymbol{\imath}\right) \boldsymbol{\imath}
$$

which implies that

$$
\imath=\frac{a-\bar{g}}{|a-\bar{g}|}
$$

Therefore

$$
\boldsymbol{a}-\overline{\boldsymbol{g}}=2 \frac{c_{1}}{m}\left|\boldsymbol{v}_{a}\right|\left(\boldsymbol{v}_{a} .(\boldsymbol{a}-\overline{\boldsymbol{g}})\right) \frac{\boldsymbol{a}-\overline{\boldsymbol{g}}}{|\boldsymbol{a}-\overline{\boldsymbol{g}}|^{2}}
$$

which in turn implies that

$$
|\boldsymbol{a}-\overline{\boldsymbol{g}}|^{2}=2 \frac{c_{1}}{m}\left|\boldsymbol{v}_{a}\right|\left(\boldsymbol{v}_{a} .(\boldsymbol{a}-\overline{\boldsymbol{g}})\right)
$$

or, equivalently

$$
|\boldsymbol{a}|^{2}-2 \boldsymbol{a} \cdot \overline{\boldsymbol{g}}+|\overline{\boldsymbol{g}}|^{2}=2 \frac{c_{1}}{m}\left|\boldsymbol{v}_{a}\right|\left(\boldsymbol{v}_{a} \cdot(\boldsymbol{a}-\overline{\boldsymbol{g}})\right)
$$

By replacing $\boldsymbol{a}$ and $|\boldsymbol{a}|^{2}$ by their expressions (35) and (36) in the above equality, one gets

$$
\ddot{s}^{2}+2 b(X, t) \ddot{s}+c(X, t)=0
$$

with

$$
\begin{aligned}
b:= & \left(\frac{\left(c_{0}+c_{1}\right)}{m}\left|\boldsymbol{v}_{a}\right| \boldsymbol{v}_{a}-\boldsymbol{g}\right) \cdot \boldsymbol{u} \\
c:= & 2 \dot{s}^{2}\left(\frac{\left(c_{0}+c_{1}\right)}{m}\left|\boldsymbol{v}_{a}\right| \boldsymbol{v}_{a}-\boldsymbol{g}\right) \cdot \boldsymbol{h}+|\overline{\boldsymbol{g}}|^{2}+\dot{s}^{4}|\boldsymbol{h}|^{2} \\
& +2 \frac{c_{1}}{m}\left|\boldsymbol{v}_{a}\right|\left(\boldsymbol{v}_{a} \cdot \overline{\boldsymbol{g}}\right)
\end{aligned}
$$

It is not difficult to verify that, of the two solutions to this quadratic equation in $\ddot{s}$, only the one adding the squared-root discriminant is physically pertinent. The function $g_{s}$ involved in the equation (34) is thus

$$
g_{s}:=-b+\sqrt{b^{2}-c}
$$

Remark: Once $s(t), \dot{s}(t), \ddot{s}(t)$ and the glider's position at the time-instant $t$ are known, the glider's orientation, i.e. the frame vectors $(\boldsymbol{\imath}, \boldsymbol{\jmath}, \boldsymbol{k})(t)$, can also be numerically determined. Indeed, $\boldsymbol{\imath}(t)$ is given by $(38)$ and $\boldsymbol{\jmath}(t)=\frac{\boldsymbol{\imath}(t) \times \boldsymbol{v}_{a}(t)}{\left|\boldsymbol{\imath}(t) \times \boldsymbol{v}_{a}(t)\right|}$ because this latter vector is orthogonal to both $\boldsymbol{\imath}(t)$ and $\boldsymbol{v}_{a}(t)$ in the case of a balanced flight. The third vector is then the cross product of the other two vectors, i.e. $\boldsymbol{k}(t)=\boldsymbol{\imath}(t) \times \boldsymbol{\jmath}(t)$. 


\section{B. Application to a Rayleigh cycle}

The path $\mathcal{C}$ is the inclined circle evoked in Section III and one may arbitrarily assume that its center is the origin of the inertial frame $\mathcal{I}$. One may also choose the glider's initial position at the top-end of the circle, i.e. $x(0)=0, y(0)=r \cos (\theta), z(0)=r \sin (\theta)$. The initial glider speed $|\boldsymbol{v}(0)|$ can be chosen arbitrarily, but large enough to yield an average speed, when going around the circle for the first time, larger than the minimal value for which sustained dynamic soaring is possible, an estimation of which is (26). The Cartesian coordinates of the glider's CoM on the circular path are

$$
\left\{\begin{array}{l}
x(s)=-r \sin (s / r) \\
y(s)=r \cos (s / r) \cos (\theta) \\
z(s)=r \cos (s / r) \sin (\theta)
\end{array}\right.
$$

and the (continuous) wind velocity is

$$
\boldsymbol{v}_{w}(z)= \begin{cases}-v_{w} \boldsymbol{\jmath}_{0} & \text { if } z \geq \frac{\epsilon}{2} \\ -v_{w}\left(0.5+\frac{z(s)}{\epsilon}\right) \boldsymbol{\jmath}_{0} & \text { if } z \in\left(-\frac{\epsilon}{2}, \frac{\epsilon}{2}\right) \\ 0 & \text { if } z \leq-\frac{\epsilon}{2}\end{cases}
$$

with $\epsilon>0$ the thickness of the shear layer (which may be chosen arbitrarily small) and $v_{w}$ the constant wind speed above the shear layer.

\section{Compared estimation and simulation results}

Once the function $g_{s}$ associated with the inclined circular path is determined, the glider's dynamics can be numerically integrated along this path. With the parameters of Table 1 and setting the initial glider speed equal to $10 \mathrm{~m} / \mathrm{s}$, the time-evolutions of the glider inertial and air speeds are represented in Figure 6. The close resemblance of Figures 5 and 6 (similar speed growth rates and maximum dynamic soaring speeds) is a first step to the validation of the estimates worked out in Section III. Another test was to determine via simulation the minimal wind speed $\bar{v}_{w, \min }$ and the minimal average speed $\bar{v}_{\text {min }}$ of the glider for which sustained dynamic soaring is possible, in order to compare them with their estimates of Section III. The values obtained via simulation with the same glider parameters and same path are $\bar{v}_{w, \text { min }} \approx 3.28 \mathrm{~m} / \mathrm{s}$ and $\bar{v}_{\min } \approx 25 \mathrm{~m} / \mathrm{s}$. They are also close to the previously estimated values of $3.21 \mathrm{~m} / \mathrm{s}$ and $24 \mathrm{~m} / \mathrm{s}$. A third test was to change the circle's 


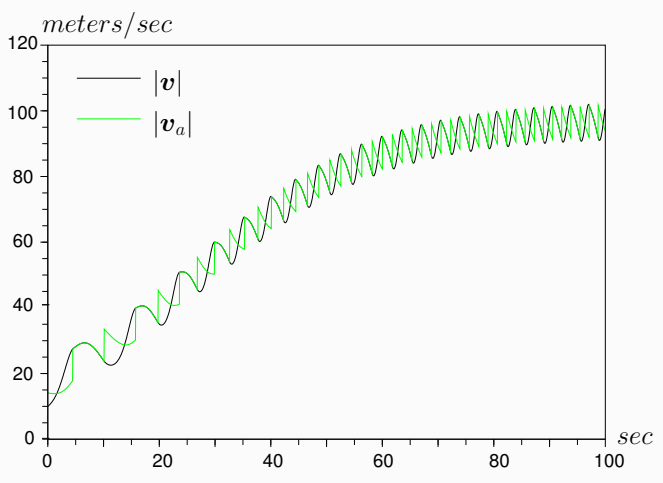

Fig. 6 Time-evolutions of the glider inertial and air speeds

angle of inclination from 0.2 rad to 0.7 rad and compare the maximal (asymptotic) average speeds $\bar{v}_{\text {max }}$ determined either via simulation or calculated from $(28)$. The values $76 \mathrm{~m} / \mathrm{s}$ and $76.9 \mathrm{~m} / \mathrm{s}$ so obtained are again close. A fourth test consisted in changing the circle's radius and verifying that the optimal radius $r_{\text {opt }}$ yielding the largest average soaring speed $\bar{v}_{\max }$ was correctly estimated by (29). Table 2 shows good concordance between simulated and estimated speeds obtained for three different radii and confirms the optimality of the radius of $47.4 m$ predicted by (29).

Table 2 Glider's average speed for different radii

\begin{tabular}{ccc}
\hline \hline radius $(m)$ & $\bar{v}_{\text {max }}(m / s$, estimated $)$ & $\bar{v}_{\text {max }}(m / s$, simulation $)$ \\
\hline 30 & 89.1 & 88.3 \\
\hline 40 & 97.25 & 96 \\
\hline 47.4 & 98.7 & 97.8 \\
\hline 50 & 98.5 & 97.1 \\
\hline 70 & 91.6 & 90.4 \\
\hline \hline
\end{tabular}

However, from the nature of the approximations used in Section III, the accuracy of the estimates should degrade when the glider speed decreases, i.e. when the wind speed decreases. To get a more precise evaluation of this degradation, we have determined by simulation and calculated from (28) the maximal glider's average speed reached with various wind speeds ranging from $5 \mathrm{~m} / \mathrm{s}$ to $25 \mathrm{~m} / \mathrm{s}$, and gathered the results in Table 3 with relative error percentages. Except for the wind speed the 
other parameters are those of Table 1.

Table 3 Accuracy of estimated soaring speeds

\begin{tabular}{cccc}
\hline \hline$v_{w}(m / s)$ & $\bar{v}_{\max }(\mathrm{m} / \mathrm{s}$, estimated $)$ & $\bar{v}_{\max }(\mathrm{m} / \mathrm{s}$, simulation $)$ & relative error $(\%)$ \\
\hline 5 & 49.3 & 48 & 2.7 \\
\hline 10 & 98.5 & 97.1 & 1.4 \\
\hline 15 & 147.8 & 146.3 & 1.0 \\
\hline 20 & 197.5 & 196 & 0.76 \\
\hline 25 & 246.3 & 245 & 0.53 \\
\hline \hline
\end{tabular}

This table confirms the loss of accuracy of the estimates for low speeds, but also shows that the accuracy remains acceptable (relative error smaller than 3\%) for a large spectrum of velocities, and becomes excellent (relative error smaller than $1 \%$ ) when the wind speed exceeds $15 \mathrm{~m} / \mathrm{s}$.

To summarize, we can assert that the estimates worked out in Section III are in good accordance with simulation results in a large spectrum of operating conditions.

\section{Application to other paths and wind profiles}

Sustained flight along a Rayleigh cycle implies the possibility of overall motion along any horizontal direction, even when this direction is opposite to the wind direction. Indeed, to this aim it suffices to slowly move the circle's center in the desired direction. This possibility is also simply simulated by adding an arbitrary small horizontal component to the wind velocity $\boldsymbol{v}_{w}=-v_{w} \boldsymbol{J}_{0}$. Now, another interest of simulation is to allow for testing operating conditions other than those associated with the Rayleigh cycle considered in Section III. Two examples illustrating this possibility are reported next.

\section{Closed eight-shape Lissajous curve}

Coordinates of the running point $P$ on this type of path are of the form

$$
x=a_{1} \sin (\phi), y=a_{2} \sin (2 \phi) \cos (\theta), z=a_{2} \sin (2 \phi) \sin (\theta)
$$




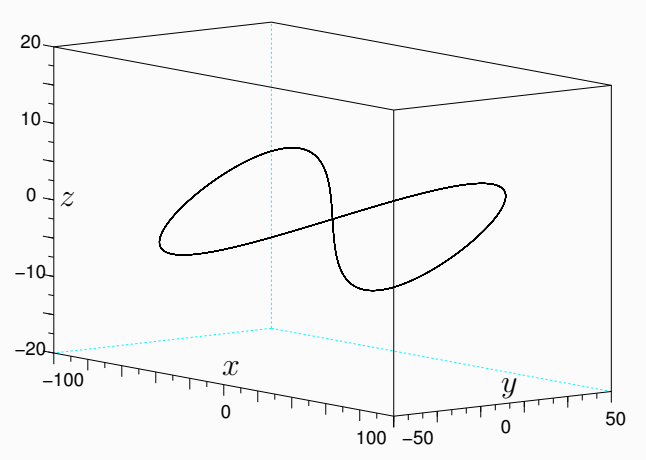

Fig. 7 Inclined Lissajous curve

with $a_{1}$ and $a_{2}$ the parameters that delimit the dimensions of this planar curve, $\phi(s) \in \mathbb{R}$, and $\theta$ the angle of inclination of the curve w.r.t. the horizontal plane. Figure 7 shows this curve in the case where $a_{1}=80 \mathrm{~m}, a_{2}=30 \mathrm{~m}$, and $\theta=0.2 \mathrm{rad}$. Differentiating these coordinates w.r.t. the curvilinear abscissa yields

$$
\begin{aligned}
& x^{\prime}(s)=a_{1} \cos (\phi(s)) \phi^{\prime}(s) \\
& y^{\prime}(s)=2 a_{2} \cos (2 \phi(s)) \cos (\theta) \phi^{\prime}(s) \\
& z^{\prime}(s)=2 a_{2} \cos (2 \phi(s)) \sin (\theta) \phi^{\prime}(s)
\end{aligned}
$$

Because $1=x^{\prime}(s)^{2}+y^{\prime}(s)^{2}+z^{\prime}(s)^{2}$ one deduces that $\phi^{\prime}(s)= \pm 1 / \sqrt{a_{1}^{2} \cos ^{2}(\phi(s))+4 a_{2}^{2} \cos ^{2}(2 \phi(s))}$, with the sign chosen according to the desired direction of motion along the curve. The unit vector tangent to the curve at the point $P$ is $\boldsymbol{u}(s)=x^{\prime}(s) \boldsymbol{\imath}_{0}+y^{\prime}(s) \boldsymbol{\jmath}_{0}+z^{\prime}(s) \boldsymbol{k}_{0}$.

The other vector $\boldsymbol{h}(s)=x^{\prime \prime}(s) \boldsymbol{\imath}_{0}+y^{\prime \prime}(s) \boldsymbol{J}_{0}+z^{\prime \prime}(s) \boldsymbol{k}_{0}$ needed to calculate the functions $b(X, t)$, $c(X, t)$ and $g_{s}(X, t)$ is obtained by differentiating the coordinates of $P$ a second time, i.e. by using

$$
\begin{aligned}
& x^{\prime \prime}(s)=a_{1}\left(-\sin (\phi(s)) \phi^{\prime}(s)^{2}+\cos (\phi(s)) \phi^{\prime \prime}(s)\right. \\
& y^{\prime \prime}(s)=2 a_{2}\left(-2 \sin (2 \phi(s)) \phi^{\prime}(s)^{2}+\cos (2 \phi(s)) \phi^{\prime \prime}(s)\right) \cos (\theta) \\
& z^{\prime \prime}(s)=2 a_{2}\left(-2 \sin (2 \phi(s)) \phi^{\prime}(s)^{2}+\cos (2 \phi(s)) \phi^{\prime \prime}(s)\right) \sin (\theta)
\end{aligned}
$$

with $\phi^{\prime \prime}(s)= \pm\left(\left(0.5 a_{1}^{2} \sin (2 \phi(s))+4 a_{2}^{2} \sin (4 \phi(s))\right) \phi^{\prime}(s)^{4}\right.$.

In this case, because the coordinates $x, y$, and $z$ are known functions of $\phi$, it suffices to define 
the state vector $X:=(\phi, s, \dot{s})$ and calculate $X(t)$ via numerical integration of the ODE

$$
\dot{X}=\left(\begin{array}{ccc}
0 & 0 & \phi^{\prime}(s) \\
0 & 0 & 1 \\
0 & 0 & 0
\end{array}\right) X+\left(\begin{array}{c}
0 \\
0 \\
g_{s}(X, t)
\end{array}\right)
$$

For the glider parameters of Table 1, a two-layer wind model with thin shear layer at $z=0$, $\boldsymbol{v}_{w}=-v_{w} \boldsymbol{J}_{0}$ with $v_{w}=10 \mathrm{~m} / \mathrm{s}$ above the shear boundary, and the Lissajous curve parameters $a_{1}=80 \mathrm{~m}, a_{2}=30 \mathrm{~m}, \theta=0.2 \mathrm{rad}$, the glider's asymptotic average speed obtained in simulation is $\bar{v}_{\max }=83 \mathrm{~m} / \mathrm{s}$. A slightly faster speed of $85 \mathrm{~m} / \mathrm{s}$ is obtained with $a_{1}=100 \mathrm{~m}, a_{2}=40 \mathrm{~m}$, and a slower speed of $74 \mathrm{~m} / \mathrm{s}$ is obtained with $a_{1}=60 \mathrm{~m}, a_{2}=25 \mathrm{~m}$. Comparison of these speeds with those obtained on a circular path tends to indicate that this latter path is slightly more energy-efficient than the eight-shape Lissajous path.

\section{Open sinusoidal path}

In order to move in some desired direction without making a loop one may consider a sinusoidal open path centered on this direction. An example of such a path is the curve parametrized by the $x$ coordinate of $P$ defined by

$$
y=r \cos (x / r) \cos (\theta), z=r \cos (x / r) \sin (\theta)
$$

with $r>0$ and $\theta$ denoting again the angle of inclination of the path w.r.t. the horizontal plane (see Figure 8 for which $r=50 \mathrm{~m}, \theta=0.2 \mathrm{rad}$ ). To simulate wind soaring along this path one first needs to determine the variation of $x$ w.r.t. the variation of the curvilinear abscissa $s$, i.e. $x^{\prime}(s)$. This is obtained by using the equality

$$
\begin{aligned}
d s^{2} & =d x^{2}+d y^{2}+d z^{2} \\
& =d x^{2}\left(1+\left(\frac{d y}{d x}\right)^{2}+\left(\frac{d z}{d x}\right)^{2}\right) \\
& =d x^{2}\left(1+\sin ^{2}(x / r)\right)
\end{aligned}
$$

and choosing one of the two solutions for $x^{\prime}=\frac{d x}{d s}$ depending on the chosen variation of $x$ w.r.t. the curvilinear abscissa. For instance, if $x$ must increase with $s$ so that $x^{\prime}$ must be positive, then

$$
x^{\prime}(s)=1 / \sqrt{1+\sin ^{2}(x(s) / r)}
$$




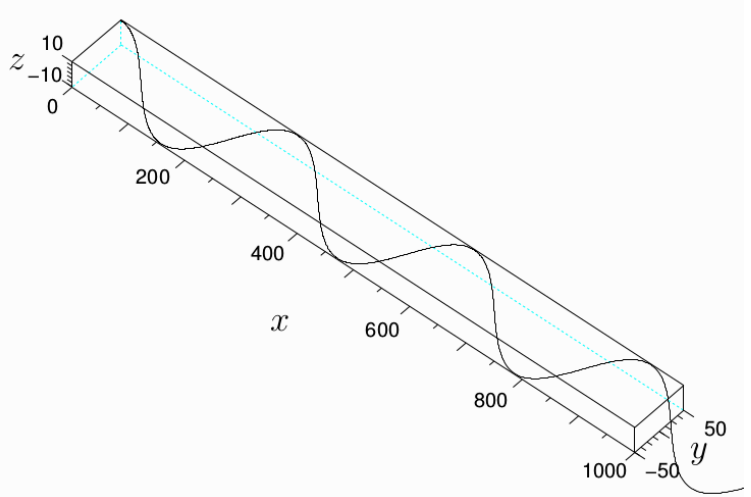

Fig. 8 Inclined sinusoidal curve

The other two coordinate derivatives w.r.t the curvilinear abscissa are then

$$
\begin{aligned}
& y^{\prime}(s)=\frac{d y}{d x} x^{\prime}(s)=-1 / \sqrt{1+\sin ^{2}(x(s) / r)} \sin (x / r) \cos (\theta) \\
& z^{\prime}(s)=\frac{d z}{d x} x^{\prime}(s)=-1 / \sqrt{1+\sin ^{2}(x(s) / r)} \sin (x / r) \sin (\theta)
\end{aligned}
$$

From there one determines the vector $\boldsymbol{h}(s):=\left(x^{\prime \prime}(s) \boldsymbol{\imath}_{0}+y^{\prime \prime}(s) \boldsymbol{J}_{0}+z^{\prime \prime}(s) \boldsymbol{k}_{0}\right)$ and, given the wind profile $\boldsymbol{v}_{w}(x, y, z, t)$, the functions $b(X, t), c(X, t)$, and $g_{s}(X, t)$. In this case, because $y$ and $z$ are functions of $x$, it is sufficient to work with the three-dimensional state vector $X=(x, s, \dot{s})^{\top}$ and calculate $X(t)$ via numerical integration of the ODE

$$
\dot{X}=\left(\begin{array}{ccc}
0 & 0 & x^{\prime}(s) \\
0 & 0 & 1 \\
0 & 0 & 0
\end{array}\right) X+\left(\begin{array}{c}
0 \\
0 \\
g_{s}(X, t)
\end{array}\right)
$$

Table 4 shows the average asymptotic velocity $\bar{v}_{\max }$ obtained with the glider parameters of Table 1 , a path inclination angle $\theta=0.2 \mathrm{rad}$, a thin wind shear layer with boundary at $z=0$, a constant wind speed of $10 \mathrm{~m} / \mathrm{s}$ above the shear layer, and a set of different wind directions given by $-\left(\sin (\psi) \boldsymbol{\imath}_{0}+\right.$ $\left.\cos (\psi) \boldsymbol{\jmath}_{0}\right)$. 


\begin{tabular}{cc}
\hline \hline wind direction $\psi(\mathrm{rad})$ & $\bar{v}_{\text {max }}(\mathrm{m} / \mathrm{s})$ \\
\hline$\pi / 6$ & 86.5 \\
\hline$-\pi / 6$ & 68 \\
\hline$\pi / 4$ & 80 \\
\hline$-\pi / 4$ & 50 \\
\hline$\pi / 3.3$ & 68.5 \\
\hline$-\pi / 3.3$ & 32.5 \\
\hline$-\pi / 2.6$ & 58 \\
\hline \hline
\end{tabular}

This table implicitly indicates that sustained flight going "up" or "down" the wind is possible when $\psi \in\left[\psi_{\min }, \psi_{\max }\right]$ with extremal angles $\psi_{\min }=-\frac{\pi}{2.6} \mathrm{rad}$ and $\psi_{\max }=\frac{\pi}{3.3} \mathrm{rad}$. For angles outside this interval we observed that sustained flight was no longer possible. Different glider parameters and wind speeds would of course yield other extremal angles. In particular, and as expected, faster wind speeds yield larger direction angle intervals for which sustained flight can be maintained. This table also indicates that the fastest average velocity of the glider is obtained when the wind direction is orthogonal to the overall path direction.

One may also test in simulation wind profiles that differ from the two-layer wind model considered so far. An example is the so-called logistic wind profile in the form

$$
v_{w}(z)=\frac{v_{w, \max }}{1+\exp \left(-\left(z-z_{0}\right) / \delta\right)}
$$

considered, for instance, in [11]. When $\delta \ll 1$ and $z_{0}=0$ this model tends to the thin shear layer model considered in Section III. This model, being differentiable w.r.t. the altitude, does not involve strict layer boundaries. Nevertheless, it may be approximated by a linear two-layer model whose shear layer is centered at $z=z_{0}$, with a thickness $\epsilon=4 \delta$ and wind speed above the shear layer equal to $v_{w, \max }$. Table 5 shows the average asymptotic velocity $\bar{v}_{\max }$ obtained with this wind profile centered at $z_{0}=0$ for different values of $\delta$, the wind direction being orthogonal to the general direction of motion, i.e. $\boldsymbol{v}_{w}=-v_{w}(z) \boldsymbol{j}_{0}$. 


\begin{tabular}{cc}
\hline \hline$\delta(m)$ & $\bar{v}_{\text {max }}(m / s)$ \\
\hline \hline 1 & 85.5 \\
\hline 2.5 & 78.5 \\
\hline 5 & 57 \\
\hline \hline
\end{tabular}

As expected the glider's performance in terms of velocity decreases when $\delta$ increases. For small values of $\delta$ the performance is similar to the one obtained with a wind two-layer model with thin shear layer.

Another popular wind profile is logarithmic and of the form

$$
v_{w}(z)=v_{w, \text { ref }} \frac{\log \left(\left(z-z_{\text {min }}+z_{1}\right) / z_{0}\right)}{\log \left(h_{\text {ref }} / z_{0}\right)}
$$

with $z_{1}\left(\geq z_{0}\right)$ denoting the glider's lowest altitude above the sea, and $h_{r e f}\left(\geq z_{1}\right)$ the glider's altitude for which $v_{w}=v_{w, r e f}$. In [2] Sachs uses this profile with $z_{0}=0.03 m, z_{1}=1.5 m, h_{r e f}=10 m$, and applies trajectory optimization software to determine that, for an albatross weighting $8.5 \mathrm{~kg}$ with glide ratio equal to 20 , an energy-neutral DS trajectory requires a minimum shear wind strength $v_{w, r e f}=8.6 \mathrm{~m} / \mathrm{s}$. For these wind profile values, the glider's parameters of Table 1, and the sinusoidal path previously considered, one can observe from simulation that a sustained flight is not possible whatever the general path direction w.r.t. the wind direction. A sustained flight in the most favorable path direction, i.e. leeward with $\psi \approx-0.5 \mathrm{rad}$, requires either a stronger wind velocity $v_{w, \text { ref }}>10.5 \mathrm{~m} / \mathrm{s}$, or using a smaller value of $z_{1}(<0.55 \mathrm{~m})$, or adaptation (optimization) of the flight-path parameters by taking, for instance, $r=25 \mathrm{~m}$ and $\theta=0.5 \mathrm{rad}$.

A better comparison with Sachs results requires to simulate the dynamics of a glider with flying characteristics close to those of an albatross. For instance, setting $m=9 \mathrm{~kg}, c_{0}=0.01$, and $\bar{c}_{0}=18$ yields, by application of (11) and (12), a glide ratio equal to 21.21 and a gliding speed of $14.4 \mathrm{~m} / \mathrm{s}$ that are close to values attributed to an average male albatross $[2,21]$. Considering an inclined sinusoidal trajectory with $r=17 \mathrm{~m}, \theta=0.5 \mathrm{rad}$ and leeward direction $\psi=-0.5 \mathrm{rad}$, and using the previously mentioned logarithmic wind profile with $z_{0}=0.03 \mathrm{~m}, z_{1}=0.75 \mathrm{~m}$ (the minimum glider's 
altitude above the sea), and $h_{r e f}=10 \mathrm{~m}$, one finds that the minimum (resp. maximum) wind-speed at the lowest (resp. highest) point on the path is equal to $0.55 v_{w, \text { ref }}$ (resp. $1.09 v_{w, \text { ref }}$ ). By Simulating this glider along this path and with this wind profile, one observes from simulation that sustained DS flight requires to use $v_{w, r e f} \geq 9.1 \mathrm{~m} / \mathrm{s}$ in the expression of the wind profile. With the minimum value of $9.1 \mathrm{~m} / \mathrm{s}$, the wind speed varies from $5.04 \mathrm{~m} / \mathrm{s}$ to $9.93 \mathrm{~m} / \mathrm{s}$ between the lowest and highest points on the path. The glider's speed varies between $10.8 \mathrm{~m} / \mathrm{s}$ and $27.2 \mathrm{~m} / \mathrm{s}$, the load factor $\frac{\left|\boldsymbol{F}_{L}\right|}{m g_{0}}$ varies from 0.9 to 4.4 , and the period for traveling one path's cycle is about $7.2 s$. These values are in the range of those observed by Pennycuick [21] for albatrosses. A smaller value of the wind strength $v_{w, r e f}$ would be obtained by further optimizing the path shape, but it is not clear at this point that the gain would be important. This latter issue, related to DS "sensitivity w.r.t. path optimization", has not (to our knowledge) been thoroughly addressed and would deserve to be further explored. A perhaps sounder reason for modifying the path shape concerns the limitation of the load factor to a maximum value, as imposed (or approximately imposed via the limitation of the lift coefficient $\left.C_{L}\right)$ in most albatross trajectory optimization studies $[2,3,6,10]$.

\section{Concluding remarks}

In this paper dynamic soaring is studied on the basis of a nonlinear point-mass flight dynamics model previously used for the design of scale-model aircraft autopilots. This model is first used to informally explain the energy-harvesting process involved in dynamic soaring and determine, via calculus approximations, estimates of various variables involved in energy neutral circular paths crossing a thin wind shear layer, i.e. so-called Rayleigh cycles. We then show that, given i) a set of parameters characterizing the flight properties of a glider, ii) a gliding path specified in terms of its curvilinear abscissa, and iii) a wind profile specifying the wind's strength and direction at any point, this model yields dynamic equations on the path that can be written as a closed-form finite-dimensional ODE amenable to standard numerical integration. This property in turn infers the possibility of simulating dynamic soaring easily for a large variety of operating conditions. This simulation facility is then used to verify the validity of the aforementioned estimates in the case of circular paths. It is also illustrated by considering two other types of paths (eight-shaped 
Lissajous curves and sinusoidal open curves) and two models of wind profile over the ocean (logisticexponential and logarithmic) proposed in several contributions studying albatrosses dynamic soaring abilities.

As pointed out in the introduction, this study departs from other engineering-oriented studies that focus on the calculation of optimal trajectories for dynamic soaring. Indeed, the first application of the proposed simulation methodology is to test if a given glider will stay aloft indefinitely by taking advantage of dynamic soaring, given a wind profile and a pre-specified trajectory that the glider has to follow. Solving a constrained optimal control problem requires important computational power and efficient dedicated programs, whereas the aforementioned test only requires using a standard numerical integration program and demands much less computational power. The two points of view are thus different. Nevertheless, they are also complementary. They both serve to evaluate the possibilities offered by dynamic soaring.

Finally, let us mention that a practical interest of testing dynamic soaring along pre-specified, not necessarily optimal, paths resides in the existence of controllers (autopilots) capable of stabilizing a (motorized) scale-model glider on such a path [7, 12, 14, 17, 18].

\section{References}

[1] Lord Rayleigh, "The soaring of birds," Nature, Vol. 27, No. 701, 1883, pp. 534-535.

[2] Sachs, G., "Minimum shear wind strenght required for dynamic soaring of albatrosses," Ibis, Vol. 147, 2005, pp. 1-10, doi:10.1111/j.1474-919x.2004.00295.x.

[3] Bonnin, V., From albatross to long range UAV flight by dynamic soaring, Ph.D. thesis, University of the West of England, http://eprints.uwe.ac.uk/26931/, 2016.

[4] Sachs, G., "Minimaler windbedarf für den dynamischen segelflug der albatrosse," Journal für Ornithologie, Vol. 134, 1993, pp. 435-445.

[5] Barnes, J., "How flies the albatross-The flight mechanics of dynamic soaring," SAE Technical paper, Vol. 2004-01-3088, doi:10.4271/2004-01-3088.

[6] Lissaman, P., "Wind energy extraction by birds and flight vehicles," Technical Soaring, Vol. 31, No. 2, 2007, pp. 52-60. 
[7] Langelaan, J., "Gust energy extraction for mini- and micro-unhabited aerial vehicles," Journal of Guidance, Control, and Dynamics (JGCD), Vol. 32, No. 2, 2009, pp. 464-473, doi:10.2514/1.37735.

[8] Deitter, M., Richards, A., Toomer, C., and Pipe, A., "Engineless uinmanned aerial vehicle propulsion by dynamic soaring," J. of Guidance, Control, and Dynamics, Vol. 32, No. 5, doi:10.2514/1.43270.

[9] Sukumar, P. and Selig, M., "Dynamic Soaring of Sailplanes over Open Fields," Journal of Aircraft, Vol. 50, No. 5, 2013, pp. 1420-1430, doi:10.2514/1.C031940.

[10] Bower, G., Boundary layer dynamic soaring for autonomous aircraft: design and validation, Ph.D. thesis, Stanford University, http://purl.stanford.edu/hp877by7094, 2011.

[11] Bousquet, G., Triantafyllou, M., and Slotine, J.-J., "Dynamic soaring in finite-thickness wind shears: an asymptotic solution," in "Proc. AIAA Guidance, Navigation, and Control Conference," Grapevine, Texas, 2017, doi:10.2514/6.2017-1908.

[12] Patel, C. and Kroo, I., "Control law design for improving UAV performance using wind turbulence," in "Proc. 44th AIAA Aerospace Sciences Meeting and Exhibit," Reno, Nevada, 2006, pp. 1-10, doi:10.2514/6.2006-231.

[13] Patel, C., Energy extraction from atmospheric turbulence to improve aircraft performance, VDM Verlag DR. Müller, 2008.

[14] Bird, J. and Langelaan, J., "Closing the loop in dynamic soaring," in "Proc. AIAA guidance, Navigation, and Control Conference," National Harbor, Maryland, 2014, pp. 1-19, doi:10.2514/6.2014-0263.

[15] Hua, M.-D., Pucci, D., Hamel, T., Morin, P., and Samson, C., "A novel approach to the automatic control of scale model airplanes," in "in Proc. of Decision and Control Conference (CDC)," , 2014.

[16] Kai, J. M., Hamel, T., and Samson, C., "A nonlinear approach to the control of a disc-shaped aircraft," in "2017 IEEE 56th Annual Conference on Decision and Control (CDC)," , 2017, pp. 2750-2755, doi:10.1109/CDC.2017.8264058.

[17] Kai, J.-M., Hamel, T., and Samson, C., "A nonlinear global approach to scale-model aircraft path following," arXiv:1803.05184, submitted to Automatica.

[18] Kai, J., Hamel, T., and Samson, C., "Design and experimental validation of a new guidance and flight control system for scale-model airplanes," in "submitted for presentation at the 57th Conf. on Decision 
and Control (CDC 2018)," Miami, Florida, 2018.

[19] Richardson, P., "High-speed dynamic soaring," R/C Soaring Digest, Vol. 29, April, 2012, pp. 36-49.

[20] Pucci, D., Hamel, T., Morin, P., and Samson, C., "Nonlinear feedback control of axisymmetric aerial vehicles," Automatica, Vol. 53, March, 2015, pp. 72-78.

[21] Pennycuick, C., "Gust soaring as a basis for the flight of petrels and albatrosses (Procellariiformes)," Avian Science, Vol. 2, 2002, pp. 1-12.

[22] Readers interested in the adjacent path control problem may refer to [7, 8, 12, 14, 17, 18] 